\title{
Mi-Cho-Coq, a framework for certifying Tezos Smart Contracts
}

\author{
Bruno Bernardo, Raphaël Cauderlier, Zhenlei Hu, Basile Pesin, and Julien \\ Tesson \\ Nomadic Labs, Paris, France \\ \{first_name.last_name\}@nomadic-labs.com
}

\begin{abstract}
Tezos is a blockchain launched in June 2018. It is written in OCaml and supports smart contracts. Its smart contract language is called Michelson and it has been designed with formal verification in mind. In this article, we present Mi-Cho-Coq, a Coq framework for verifying the functional correctness of Michelson smart contracts. As a case study, we detail the certification of a Multisig contract with the Mi-Cho-Coq framework.
\end{abstract}

Keywords: Certified programming $\cdot$ Programming languages $\cdot$ Blockchains - Smart contracts.

\section{Introduction to Tezos}

Tezos is a public blockchain launched in June 2018. It is mostly implemented in OCaml [18] and its code is open source [3. Like Ethereum, Tezos is an account based smart contract platform. This section is a high-level broad overview of Tezos to distinguish it from similar projects like Bitcoin and Ethereum.

Consensus algorithm Unlike Bitcoin and Ethereum, Tezos' consensus algorithm is based on a Proof-of-Stake algorithm [2]: rights to produce new blocks are given to accounts that own a stake. More precisely, there is a delegation mechanism and the block-producing rights of each account are given in probabilistic proportion to the number of tokens that have been delegated to this account. Block producers have to make a security deposit that is slashed if their behaviour looks malicious, for example if they produce two different blocks for the same level (double spending attack).

On-chain voting Another key point of Tezos is its on-chain governance mechanism. The codebase can be changed by a vote of the token holders via their delegates. This helps preventing divisions amongst the community that could lead to forks. Only a delimited part of the codebase, named the economic ruleset or the economic protocol [15]16, can be changed. This part contains the rules that define what a valid transaction is, what a valid block is, as well 
as how to choose between multiple chains. Thus, the economic ruleset contains, amongst other things, the consensus algorithm, the language for smart contracts and also the voting rules [4. It does not contain the network and storage layers. If a proposal is accepted, nodes need not to stop and restart: the new code is downloaded from other peers, dynamically compiled and hot swapped. At the moment, the voting procedure lasts approximately three months but that could be changed in the future via a vote.

Focus on formal verification Our long-term ambition is to have certified code in the whole Tezos codebase 11 as well as certified smart contracts. The choice of OCaml as an implementation language is an interesting first step: OCaml gives Tezos good static guarantees since it benefits from OCaml's strong type system and memory management features. Furthermore, formally verified OCaml code can be produced by a variety of tools such as $\mathrm{F}^{*}$ [22], Coq [23], Isabelle/HOL [19, Why3 [14, and FoCaLiZe [20]. Another specificity of Tezos is the use of formally verified cryptographic primitives. Indeed the codebase uses the HACL* library 24], which is certified C code extracted from an implementation of Low*, a fragment of $\mathrm{F}^{*}$. This article presents Mi-Cho-Coq, a framework for formal verification of Tezos smart contracts, written in the Michelson programming language. It is organised as follows: Section 2 gives an overview of the Michelson smart contract language, the Mi-Cho-Coq framework is then presented in Section 3, a case study on a Multisig smart contract is then conducted in Section 4. Section 5 presents some related workd and finally Section 6 concludes the article by listing directions for future work.

The Mi-Cho-Coq framework, including the Multisig contract described in Section 4 is available at https://gitlab.com/nomadic-labs/mi-cho-coq/tree/FMBC_2019.

\section{Overview of Michelson}

Smart contracts are Tezos accounts of a particular kind. They have a private access to a memory space on the chain called the storage of the smart contract, each transaction to a smart contract account contains some data, the parameter of the transaction, and a script is run at each transaction to decide if the transaction is valid, update the smart contract storage, and possibly emit new operations on the Tezos blockchain.

Michelson is the language in which the smart contract scripts are written. The Michelson language has been designed before the launch of the Tezos blockchain. The most important parts of the implementation of Michelson, the typechecker and the interpreter, belong to the economic ruleset of Tezos so the language can evolve through the Tezos amendment voting process.

\footnotetext{
${ }^{1}$ Note that since code changes must be approved by the Tezos community, we can only propose a certified implementation of the economic ruleset.
} 


\subsection{Design rationale}

Smart contracts operate in a very constrained context: they need to be expressive, evaluated efficiently, and their resource consumption should be accurately measured in order to stop the execution of programs that would be too greedy, as their execution time impacts the block construction and propagation. Smart contracts are non-updatable programs that can handle valuable assets, there is thus a need for strong guarantees on the correctness of these programs.

The need for efficiency and more importantly for accurate account of resource consumption leans toward a low-level interpreted language, while the need for contract correctness leans toward a high level, easily auditable, easily formalisable language, with strong static guarantees.

To satisfy these constraints, Michelson was made a Turing-complete, low level, stack based interpreted language ( $\grave{a}$ la Forth), enabling the resource measurement, but with some high level features à la ML: polymorphic products, options, sums, lists, sets and maps data-structures with collection iterators, cryptographic primitives and anonymous functions. Contracts are pure functions that take a stack as input and return a stack as output. This side-effect free design is an asset for the conception of verification tools.

The language is statically typed to ensure the well-formedness of the stack at any point of the program. This means that if a program is well typed, and if it is being given a well-typed stack that matches its input expectation, then at any point of the program execution, the given instruction can be evaluated on the current stack.

Moreover, to ease the formalisation of Michelson, ambiguous or hidden behaviours have been avoided. In particular, unbounded integers are used to avoid arithmetic overflows and division returns an option (which is None if and only if the divisor is 0) so that the Michelson programmer has to specify the behaviour of the program in case of division by 0; she can however still explicitly reject the transaction using the FAILWITH Michelson instruction.

\subsection{Quick tour of the language}

The full language syntax, type system, and semantics are documented in [1], we give here a quick and partial overview of the language.

Contracts' shape A Michelson smart contract script is written in three parts: the parameter type, the storage type, and the code of the contract. A contract's code consists in one block of code that can only be called with one parameter, but multiple entry points can be encoded by branching on a nesting of sum types and multiple parameters can be paired into one.

When the contract is deployed (or originated in Tezos lingo) on the chain, it is bundled with a data storage which can then only be changed by a contract successful execution. The parameter and the storage associated to the contract are paired and passed to the contract's code at each execution, it has to return a list of operations and the updated storage. 
Seen from the outside, the type of the contract is the type of its parameter, as it is the only way to interact with it.

Michelson Instructions As usual in stack-based languages, Michelson instructions take their parameters on the stack. All Michelson instructions are typed as a function going from the expected state of the stack, before the instruction evaluation, to the resulting stack. For example, the AMOUNT instruction used to obtain the amount in $\mu$ te $z$ of the current transaction has type 'S $\rightarrow$ mutez: 'S meaning that for any stack type 'S, it produces a stack of type mutez: 'S. Some instructions, like comparison or arithmetic operations, exhibit non-ambiguous ad-hoc polymorphism: depending on the input arguments' type, a specific implementation of the instruction is selected, and the return type is fixed. For example SIZE

has the following types: $\begin{aligned} & \text { bytes: 'S } \rightarrow \text { nat: 'S } \\ & \text { string: 'S } \rightarrow \text { nat:'S }\end{aligned} \quad$ map 'key 'val:'S $\rightarrow$ nat:'S

While computing the size of a string or an array of bytes is similarly implemented, under the hood, the computation of map size has nothing to do with the computation of string size.

Finally, the contract's code is required to take a stack with a pair parameterstorage and returns a stack with a pair operation list-storage:

(parameter_ty*storage_ty): [] $\rightarrow$ (operation list*storage_ty): [].

The operations listed at the end of the execution can change the delegate of the contract, originate new contracts, or transfer tokens to other addresses. They will be executed right after the execution of the contract. The transfers can have parameters and trigger the execution of other smart contracts: this is the only way to perform inter-contract calls.

A short example - the Vote contract. We want to allow users of the blockchain to vote for their favorite formal verification tool. In order to do that, we create a smart contract tasked with collecting the votes. We want any user to be able to vote, and to vote as many times as they want, provided they pay a small price (say 5 tez). We originate the contract with the names of a selection of popular tools: Agda, Coq, Isabelle and K_framework, which are placed in the long-term storage of the contract, in an associative map between the tool's name and the number of registered votes (of course, each tool starts with 0 votes).

In the figure 1a, we present a voting contract, annotated with the state of the stack after each line of code. When actually writing a Michelson contract, development tools (including an Emacs Michelson mode) can interactively, for any point of the code, give the type of the stack provided by the Michelson typecheck of a Tezos node.

Let's take a look at our voting program: First, the description of the storage and parameter types is given on lines 1-2. Then the code of the contract is given. On line 5, AMOUNT pushes on the stack the amount of (in $\mu$ tez) sent to the contract address by the user. The threshold amount $(5 \mathrm{tez})$ is also pushed on the stack on line 6 and compared to the amount sent: COMPARE pops the two top 


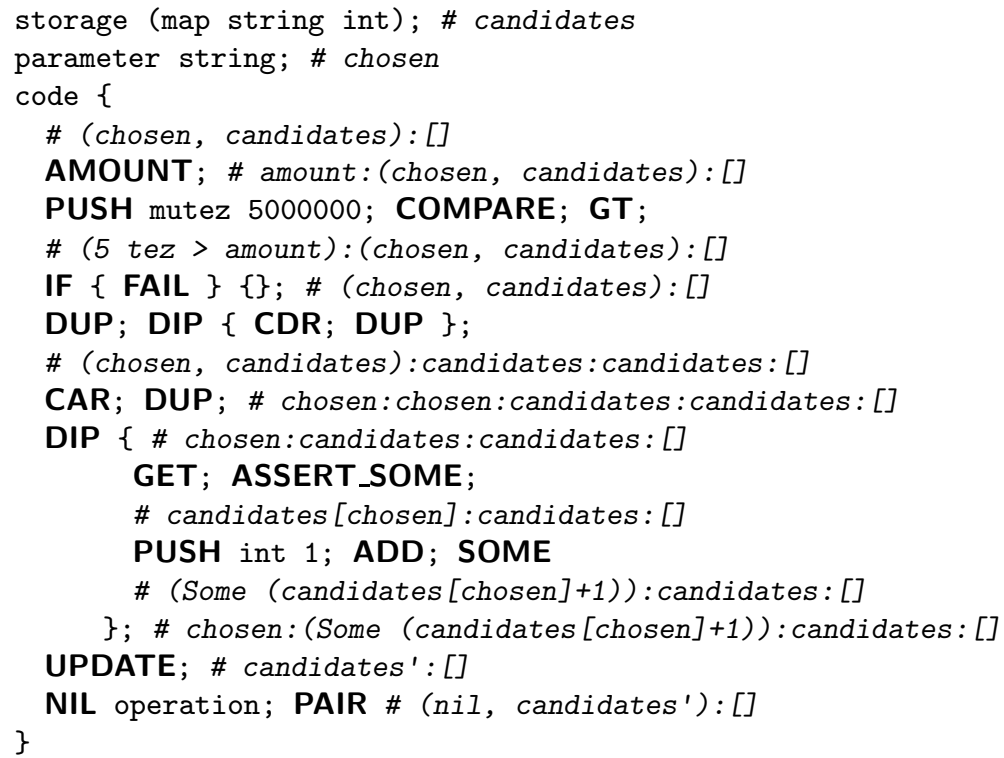

(a)

\{Elt "Agda" 0 ; Elt "Coq" 0 ; Elt "Isabelle" 0 ; Elt "K" 0\}

(b)

Fig. 1: A simple voting contract a and an example of initial storage $b$

values of the stack, and pushes either $-1,0$ or 1 depending on the comparison between the value. GT then pops this value and pushes true if the value is 1 . If the threshold is indeed greater than the required amount, the first branch of the IF is executed and FAIL is called, interrupting the contract execution and cancelling the transaction.

If the value was false, the execution continues on line 9, where we prepare the stack for the next action: DUP copies the top of the stack, we then manipulate the tail of the stack while preserving it's head using DIP: there, we take the right element of the (chosen, candidates) pair with CDR, and we duplicate it again. By closing the block guarded by DIP we recover the former stack's top, and the following line takes its left element with CAR, and duplicates it.

On line 12, we use DIP to protect the top of the stack again. GET then pops chosen and candidates from the stack, and pushes an option containing the number of votes of the candidate, if it was found in the map. If it was not found, ASSERT_SOME makes the program fail. On line 15, the number of votes is incremented by ADD, and packed into an option type by SOME.

We then leave the DIP block to regain access to value at the top of the stack (chosen). On line 18, UPDATE pops the three values remaining on top of the stack, and pushes the candidates map updated with the incremented value for 
chosen. Finally, we push an empty list of operations with NIL operation, and pair the two elements on top of the stack to get the correct return type.

\section{Mi-Cho-Coq : a Verification Framework in Coq for Michelson}

Mi-Cho-Coq consists of an implementation of a Michelson interpreter in Coq as well as a weakest precondition calculus à la Dijkstra [13].

Michelson syntax and typing in Coq Michelson's type system, syntax and semantics, as described in the main documentation, are fully formalised in MiCho-Coq.

The abstract syntax tree of a Michelson script is a term of an inductive type which carries the script type :

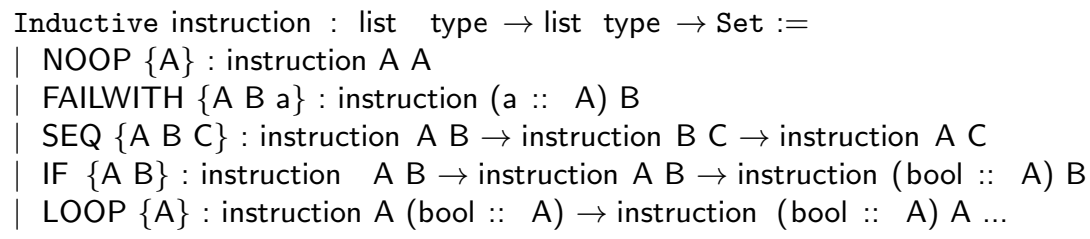

A Michelson code is usually a sequence of instructions (SEQ), which is one of the instruction constructors. It has type instruction stA stB where st $A$ and stB are respectively the type of the input stack and of the output stack.

The stack type is a list of Michelson type constructions, defined in the type inductive:

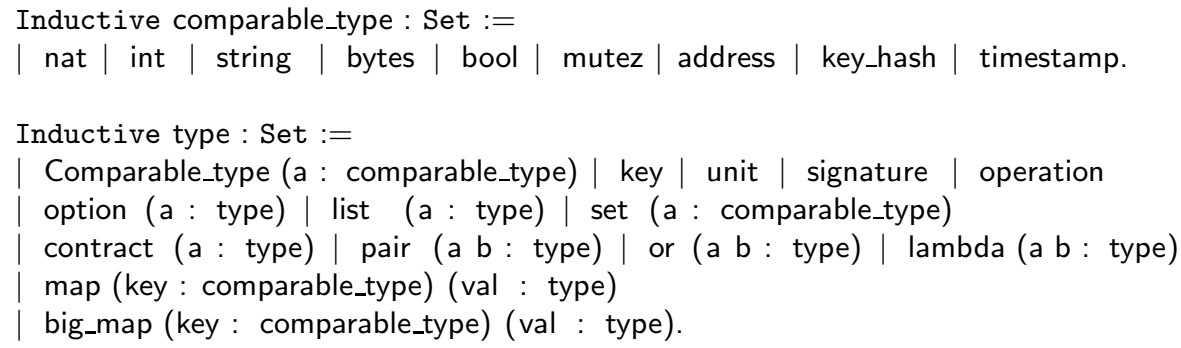

A full contract, for a given storage type storage and parameter type params is an instruction of type

instruction ((pair params storage) :: nil ) ((pair (list operation) storage ) :: nil ).

Thanks to the indexing of the instruction inductive by the input and output stack types, only well-typed Michelson instructions are representable in Mi-ChoCoq. This is very similar to the implementation of Michelson in the Tezos node which uses a similar feature in OCaml: generalised algebraic datatypes.

To ease the transcription of Michelson contracts into Mi-Cho-Coq AST we use notations so that contracts in Mi-Cho-Coq look very similar to actual Michelson 
code. The main discrepancy between Michelson and Mi-Cho-Coq syntax being that due to parsing limitations, the Michelson semi-colon instruction terminator has to be replaced by a double semi-colon instructions separator.

The ad-hoc polymorphism of Michelson instructions is handled by adding an implicit argument to the corresponding instruction constructor in Mi-ChoCoq. This argument is a structure that carries an element identifying the actual implementation of the instruction to be used. As the argument is implicit and maximally inserted, Coq type unifier tries to fill it with whatever value can fit with the known types surrounding it, i.e. the type of the input stack. Possible values are declared through the Coq's canonical structures mechanism, which is very similar to (Coq's or Haskell's) typeclasses.

Michelson interpreter in Coq Michelson semantics is formalised in Coq as an evaluator eval of type forall $\{A B$ : list type $\}$, instruction $A B \rightarrow$ nat $\rightarrow$ stack $A$ $\rightarrow \mathrm{M}$ (stack $\mathrm{B}$ ) where $\mathrm{M}$ is the error monad used to represent the explicit failure of the execution of a contract. The argument of type nat is called the fuel of the evaluator. It represents a bound on the depth of the execution of the contract and should not be confused with Michelson's cost model which is not yet formalised in Mi-Cho-Coq.

Some domain specific operations which are hard to define in Coq are axiomatised in the evaluator. These include cryptographic primitives, data serialisation, and instructions to query the context of the call to the smart contract (amount and sender of the transaction, current date, balance and address of the smart contract).

A framework for verifying smart contracts To ease the writing of correctness proofs in Mi-Cho-Coq, a weakest precondition calculus is defined as a function eval_precond of type forall \{fuel A B $\}$, instruction A B $\rightarrow$ (stack B $\rightarrow$ Prop) $\rightarrow$ (stack $A \rightarrow$ Prop) that is a Coq function taking as argument an instruction and a predicate over the possible output stacks of the instruction (the postcondition) and producing a predicate on the possible input stacks of the instruction (the precondition).

This function is proved correct with respect to the evaluator:

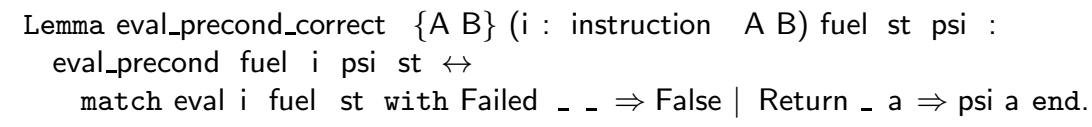

Note that the right-hand side formula is the result of the monad transformer of [6] which here yields a simple expression thanks to the absence of complex effects in Michelson.

A short example - the Vote contract We give below, as an example, a formal specification of the voting contract seen previously. We want the contract to take into account every vote sent in a transaction with an amount superior to 5 tez. Moreover, we want to only take into account the votes toward an 
actual available choice (the contract should fail if the wrong name is sent as a parameter). Finally, the contract should not emit any operation.

In the following specification, the precondition is the condition that must be verified for the contract to succeed. The postcondition fully describes the new state of the storage at the end of the execution, as well as the potentially emitted operations. amount refers to the quantity of $\mu t e z$ sent by the caller for the transaction.

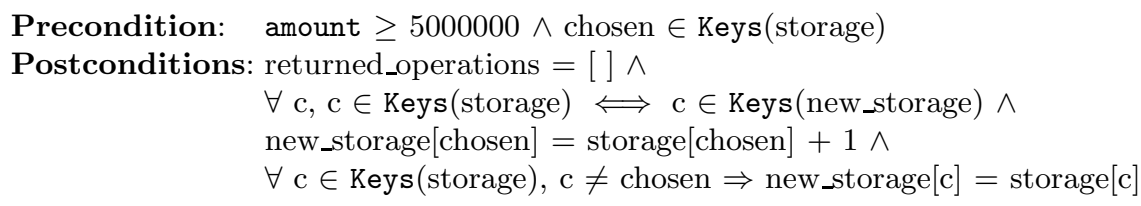

Despite looking simple, proving the correctness of the vote contract still needs a fair number of properties about the map data structure. In particular we need some lemmas about the relations between the mem, get and update functions, which we added to the Mi-Cho-Coq library to prove this contract.

Once these lemmas are available, the contract can easily be proved by studying the three different situations that can arise during the execution : the contract can fail (either because the sender has not sent enough tez or because they have not selected one of the possible candidates), or the execution can go smoothly.

\section{A case study : the Multisig Contract}

The Multisig contract is a typical example of access-control smart contract. A Multisig contract is used to share the ownership of an account between several owners. The owners are represented by their cryptographic public keys in the contract storage and a pre-defined threshold (a natural number between 1 and the number of owners) of them must agree for any action to be performed by the Multisig contract.

Agreement of an owner is obtained by requiring a cryptographic signature of the action to be performed. To ensure that this signature cannot be replayed by an attacker to authenticate in another call to a Multisig contract (the same contract or another one implementing the same authentication protocol), a nonce is appended to the operation before signing. This nonce consists of the address of the contract on the blockchain and a counter incremented at each call.

Michelson Implementation To be as generic as possible, the possible actions of our Multisig contract are:

- produce a list of operations to be run atomically

- change the threshold and the list of owner public keys

The contract features two entrypoints named default and main. The default entrypoint takes no parameter (it has type unit) and lets unauthenticated users 
send funds to the Multisig contract. The main entrypoint takes as parameters an action, a list of optional signatures, and a counter value. It checks the validity and the number of signatures and, in case of successful authentication, it executes the required action and increment the counter.

The Michelson script of the Multisig contract is available at [10]. The code of the default entrypoint is trivial. The code for the main entrypoint can be divided in three parts: the header, the loop, and the tail.

The header packs together the required action and the nonce and checks that the counter given as parameter matches the one stored in the contract.

The loop iterates over the stored public keys and the optional signatures given in parameter. It counts and checks the validity of all the signatures.

Finally the contract tail checks that the number of provided signatures is at least as large as the threshold, it increments the stored counter, and it runs the required action (it either evaluates the anonymous function passed in the contract parameter and emits the resulting operations or modifies the contract storage to update the list of owner public keys and the threshold).

Specification and Correctness Proof Mi-Cho-Coq is a functional verification framework. It is well suited to specify the relation between the input and output stacks of a contract such as Multisig but it is currently not expressive enough to state properties about the lifetime of a smart contract nor the interaction between smart contracts. For this reason, we have not proved that the Multisig contract is resistant to replay attacks. However, we fully characterise the behaviour of each call to the Multisig contract using the following specification of the Multisig contract, where env is the evaluation environment containing among other data the address of the contract (self env) and the amount of the transaction (amount env).

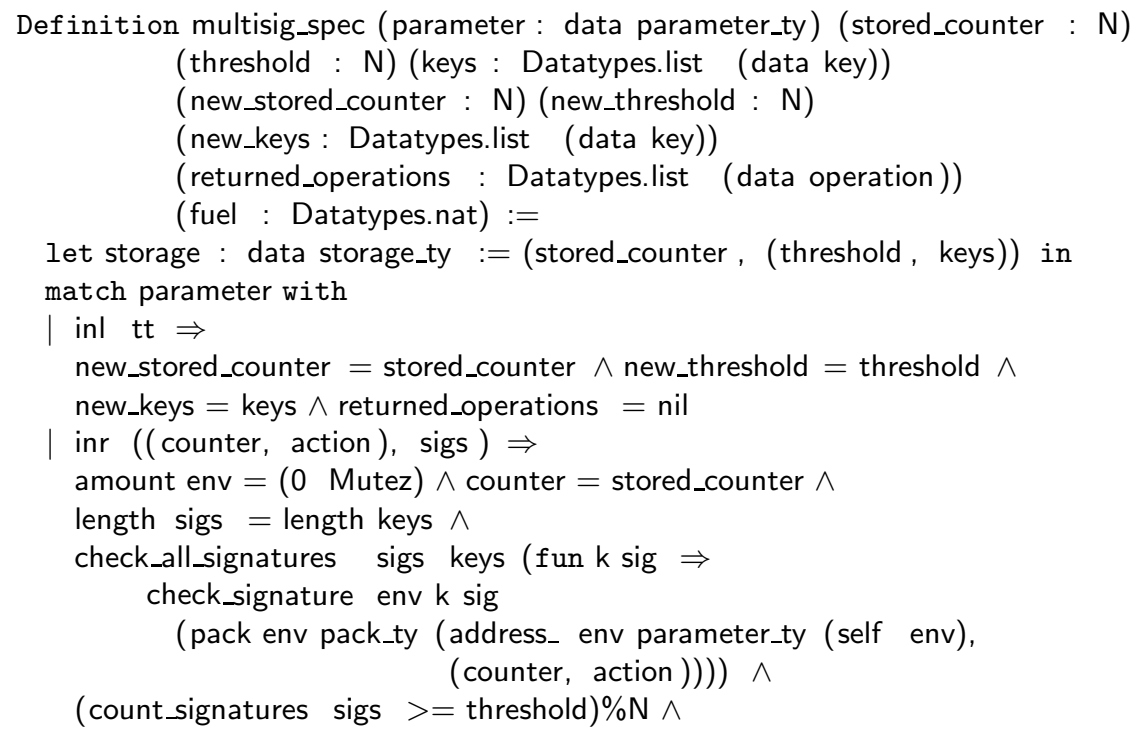




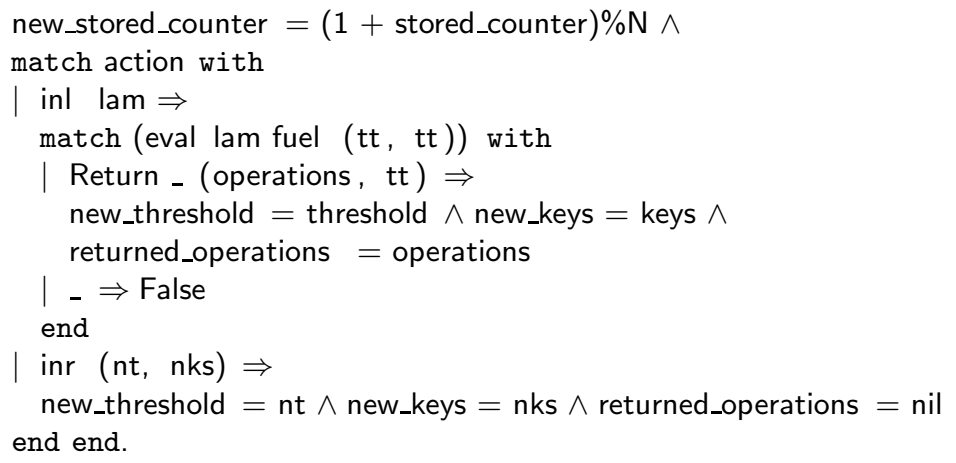

Using the Mi-Cho-Coq framework, we have proved the following theorem:

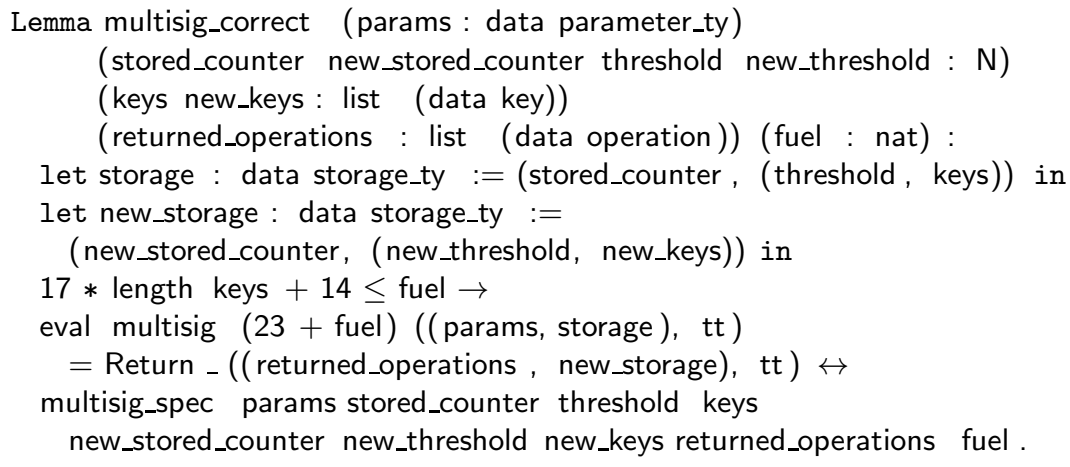

The proof relies heavily on the correctness of the precondition calculus. The only non-trivial part of the proof is the signature checking loop. Indeed, for efficiency reasons, the Multisig contract checks the equality of length between the optional signature list and the public key list only after checking the validity of the signature; an optional signature and a public key are consumed at each loop iteration and the list of remaining optional signatures after the loop exit is checked for emptiness afterward. For this reason, the specification of the loop has to allow for remaining unchecked signatures.

\section{Related Work}

Formal verification of smart contracts is a recent but active field. The $\mathrm{K}$ framework has been used to formalise [17] the semantics of both low-level and highlevel smart contract languages for the Ethereum and Cardano blockchains. These formalizations have been used to verify common smart contracts such as Casper, Uniswap, and various implentation of the ERC20 and ERC777 standards.

Ethereum smart contracts, written in the Solidity high-level language, can also be certified using a translation to the $\mathrm{F}^{*}$ dependently-typed language [8].

The Zen Protocol [5] directly uses $\mathrm{F}^{*}$ as its smart contract language so that smart contracts of the Zen Protocol can be proved directly in $\mathrm{F}^{*}$. Moreover, 
runtime tracking of resources can be avoided since computation and storage costs are encoded in the dependent types.

The Scilla 21] language of the Zilliqa blockchain has been formalised in Coq. This language is higher-level (but also less featureful) than Michelson. Its formalisation includes inter-contract interaction and contract lifespan properties. This has been used to show safety properties of a crowdfounding smart contract.

\section{$6 \quad$ Limits and Future Work}

As we have seen, the Mi-Cho-Coq verification framework can be used to certify the functional correctness of non-trivial smart contracts of the Tezos blockchain such as the Multisig contract. We are currently working on several improvements to extend the expressivity of the framework; Michelson's cost model and the semantics of inter-contract interactions are being formalised.

In order to prove security properties, such as the absence of signature replay in the case of the Multisig contract, an adversarial model has to be defined. This task should be feasible in Coq but our current plan is to use specialised tools such as Easycrypt [7] and ProVerif [9].

No code is currently shared between Mi-Cho-Coq and the Michelson evaluator written in OCaml that is executed by the Tezos nodes. We would like to raise the level of confidence in the fact that both evaluators implement the same operational semantics. We could achieve this either by proposing to the Tezos stakeholders to amend the ecomomic protocol to replace the Michelson evaluator by a version extracted from Mi-Cho-Coq or by translating to Coq the OCaml code of the Michelson evaluator using a tool such as CoqOfOCaml 12 . or CFML [11] and then prove the resulting Coq function equivalent to the MiCho-Coq evaluator.

Last but not least, to ease the development of certified compilers from highlevel languages to Michelson, we are working on the design of an intermediate compilation language called Albert that abstracts away the Michelson stack.

\section{References}

1. Michelson: the language of Smart Contracts in Tezos. http://tezos.gitlab.io/mainnet/whitedoc/michelson.html

2. Proof-of-stake in Tezos.https://tezos.gitlab.io//mainnet/whitedoc/proof_of_stake.html

3. Tezos code repository. https://gitlab.com/tezos/tezos

4. Voting in Tezos. https://tezos.gitlab.io//mainnet/whitedoc/voting.html

5. An introduction to the zen protocol. https://www.zenprotocol.com/files/zen_protocol_white_paper.pdf (2017)

6. Ahman, D., Hritcu, C., Martínez, G., Plotkin, G.D., Protzenko, J., Rastogi, A., Swamy, N.: Dijkstra monads for free. CoRR abs/1608.06499 (2016), http://arxiv.org/abs/1608.06499

7. Barthe, G., Dupressoir, F., Grégoire, B., Kunz, C., Schmidt, B., Strub, P.: Easycrypt: A tutorial. In: Foundations of Security Analysis and Design VII - FOSAD 2012/2013 Tutorial Lectures. Lecture Notes in Computer Science, vol. 8604, pp. 146-166. Springer (2013). https://doi.org/10.1007/978-3-319-10082-1_6 
8. Bhargavan, K., Delignat-Lavaud, A., Fournet, C., Gollamudi, A., Gonthier, G., Kobeissi, N., Kulatova, N., Rastogi, A., Sibut-Pinote, T., Swamy, N., ZanellaBéguelin, S.: Formal verification of smart contracts: Short paper. pp. 91-96. PLAS '16, ACM, New York, NY, USA (2016). https://doi.org/10.1145/2993600.2993611

9. Blanchet, B.: Modeling and verifying security protocols with the applied pi calculus and proverif. Foundations and Trends in Privacy and Security 1(1-2), 1-135 (2016). https://doi.org/10.1561/3300000004

10. Breitman, A.: Multisig contract in Michelson. https://github.com/murbard/smart-contracts/blob/master/multisig/michelson/generic_multisig.tz

11. Charguéraud, A.: Characteristic formulae for the verification of imperative programs. pp. 418-430. ICFP '11, ACM, New York, NY, USA (2011). https://doi.org/10.1145/2034773.2034828

12. Claret, G.: Program in Coq. Theses, Université Paris Diderot - Paris 7 (Sep 2018), https://hal.inria.fr/tel-01890983

13. Dijkstra, E.W.: Guarded commands, nondeterminacy and formal derivation of programs. Commun. ACM 18(8), 453-457 (Aug 1975). https://doi.org/10.1145/360933.360975

14. Filliâtre, J.C., Paskevich, A.: Why3 - Where Programs Meet Provers. In: ESOP'13 22nd European Symposium on Programming. LNCS, vol. 7792. Springer, Rome, Italy (Mar 2013), https://hal.inria.fr/hal-00789533

15. Goodman, L.M.: Tezos: A self-amending crypto-ledger. position paper. https://tinyurl.com/tezospp (2014)

16. Goodman, L.M.: Tezos: A self-amending crypto-ledger. white paper. https://tinyurl.com/tezoswp (2014)

17. Hildenbrandt, E., Saxena, M., Zhu, X., Rodrigues, N., Daian, P., Guth, D., Moore, B., Zhang, Y., Park, D., Ştefănescu, A., Roşu, G.: Kevm: A complete semantics of the ethereum virtual machine. In: 2018 IEEE 31st Computer Security Foundations Symposium. pp. 204-217. IEEE (2018)

18. Leroy, X., Doligez, D., Frisch, A., Garrigue, J., Rémy, D., Vouillon, J.: The OCaml system release 4.08: Documentation and user's manual. User manual, Inria (Jun 2019), http://caml.inria.fr/pub/docs/manual-ocaml/

19. Nipkow, T., Wenzel, M., Paulson, L.C.: Isabelle/HOL: A Proof Assistant for Higher-order Logic. Springer-Verlag, Berlin, Heidelberg (2002)

20. Pessaux, F.: FoCaLiZe: Inside an F-IDE. In: Workshop F-IDE 2014. Proceedings F-IDE 2014, Grenoble, France (May 2014). https://doi.org/10.4204/EPTCS.149.7 https://hal.archives-ouvertes.fr/hal-01203501

21. Sergey, I., Kumar, A., Hobor, A.: Scilla: a smart contract intermediate-level language. CoRR abs/1801.00687 (2018), http://arxiv.org/abs/1801.00687

22. Swamy, N., Hritcu, C., Keller, C., Rastogi, A., Delignat-Lavaud, A., Forest, S., Bhargavan, K., Fournet, C., Strub, P.Y., Kohlweiss, M., Zinzindohoué, J.K., Zanella-Béguelin, S.: Dependent types and multi-monadic effects in $\mathrm{F}^{*}$. In: POPL. pp. 256-270. ACM (Jan 2016), https://www.fstar-lang.org/papers/mumon/

23. The Coq development team: The Coq Reference Manual, version 8.9 (Nov 2018), http://coq.inria.fr/doc

24. Zinzindohoué, J.K., Bhargavan, K., Protzenko, J., Beurdouche, B.: Hacl*: A verified modern cryptographic library. Cryptology ePrint Archive, Report 2017/536 